Revue : Annales de géographie, nº48, mars-avril 2006, pp. 197-216

\title{
Les ressources du département et du canton dans la « petite fabrique des territoires ${ }^{1}$
}

Frédéric Tesson, maître de conférences à l'université de Pau et des Pays de l'Adour

La pression qui pèse sur le département depuis les lois $1992^{2}$ et $1995^{3}$ et surtout celles de $1999^{4}$, créant puis renforçant les Pays d'une part et généralisant la coopération intercommunale d'autre part, a largement été relayée tant par le monde de l'action que par la sphère de la recherche. L'ouvrage de L. Laurent (2002), véritable plaidoyer pour les Pays en même temps qu'épitaphe pour le département, constitue d'ailleurs un remarquable témoignage de ce consensus «anti-départementaliste». L'idée n'est pas nouvelle, on le montrera, mais on peut voir là un changement dans le fait que ce ne sont plus exclusivement les chercheurs qui viennent appuyer la parole des acteurs sur la scène médiatique voire politique, mais bien les acteurs qui utilisent les tribunes généralement réservées aux chercheurs pour affirmer et étayer leurs positions. Cet ouvrage va dans le sens du «rapport Mauroy $»^{5}$ réalisé en 2000 pour le Premier ministre L. Jospin, intitulé « refonder l'action politique locale », mais, bien que sévère à l'égard du département, celui-ci ne propose cependant pas de le rayer du paysage des circonscriptions territoriales françaises.

Cette remise en cause cyclique et cette fin promise d'une maille intermédiaire de l'espace français, en même temps que la résistance dont elle fait preuve, n'est pas sans rappeler la fin du territoire, idée qui a pris corps dans la dernière décennie du XX ${ }^{\text {ème }}$ siècle avec B. Badie (1995) et avant lui J.P. Balligand et D. Maquard (1990) pour sa version jacobine, voire X. Piolle ${ }^{6}$, dans sa dimension sociale. Cette idée semblait autoriser de nouveaux rapports à l'espace, moins continus, plus souples et nous avions mis en évidence une manifestation singulière de cette nouveauté chez l'acteur public à travers la politique des réseaux de villes (F. Tesson, 1996). Pourtant, force est de constater, là encore, que si des figures d'un type nouveau naissent, se propagent, portées par une mobilité accrue et indéniable, par un individualisme exacerbé dans une société surmoderne (M. Augé, 1992), postmoderne (J. F. Lyotard, 1979) voire hypermoderne (G. Lipovetski, 2004), le territoire, sa quête (G. Di Méo, 1998), sa construction (M. Vanier, 1995) et son modèle (J. Lévy, M. Lussault, 2003 ), en fait, son idéologie, ne cessent de hanter nos

\footnotetext{
${ }^{1}$ Je tiens à remercier : J. Alduy, A. Bleton-Ruget, J.-P. Charrié, G. Di Méo et D. Laplace pour leur relecture et/ou leur conseils

${ }^{2}$ Loi ATR du 6 février 1992

${ }^{3}$ LOADT du 4 février 1995

${ }^{4}$ LOADDT du 25 juin 1999, loi relative au renforcement et la simplification de la coopération intercommunale du 12 juillet 1999

${ }^{5} \mathrm{http}: / /$ www.alcol.org/FFL_rapdecentralisation.htm

${ }^{6} \mathrm{Cf}$ les deux colloques « la fin des territoires » organisés par X. Piolle et C. Raffestin (1994-95)
} 
représentations et de participer à la construction des valeurs de nos sociétés. Ces «prophéties », l'une comme l'autre, sont donc restées sans effet. Comme le territoire, le département est encore là et, serions-nous peut-être tentés de dire plus là que jamais. En effet, la loi constitutionnelle du 28 mars 2003 préserve au département sa place dans le processus de décentralisation, et la loi du 13 août 2004, relative aux responsabilités locales, le renforce dans de nouvelles compétences. Peut-être à la manière des prédictions destructrices évoquées par R. K. Merton (1997 [1953]), leur fin trop évidente a-t-elle suscité la mobilisation de leurs acteurs et garanti au final leur reproduction.

Département, canton, EPCI, Pays sont aujourd'hui des territoires en construction. Ce sont, pour le politique, autant d'espaces légitimes, plus ou moins anciens, plus ou moins reconnus, plus ou moins hérités mais tous en quête d'une identité, d'une reconnaissance en tant que territoire (M. Vanier, 2004). Notre propos s'attachera à comprendre l'emballement du processus de construction des territoires locaux, les mécanismes de superposition et d'enchevêtrement à l'œuvre et les ressources dont disposent le canton et le département dans cette mise en concurrence. Ce questionnement est né de l'observation empirique, appuyé par de nombreuses recherches (J.F. Gaucher et G. Baudelle, 1995, R. Le Saout et F. Madoré (dir.), 2004), du télescopage des périmètres des nouveaux territoires intercommunaux (EPCI ou Pays) avec ceux des territoires institutionnels englobants. P. A. Landel (2004) va même jusqu'à évoquer, "la capacité de nombre de conseiller généraux à modeler les contours des intercommunalités sur ceux des cantons $»(\mathrm{p} .76)$. Cette confusion de périmètre, notamment en milieu rural mais aussi parfois périurbain, nous interroge. Quels enjeux recèle ce gabarit d'EPCI ? Dans le cas d'un cumul entre les fonctions de conseiller général et de président d'EPCI, ce schéma ne porte-til pas en lui la possibilité de transformer un espace d'élection (le canton) en espace d'action (l'EPCI) ? Elu par ses pairs à la présidence de la communauté et au suffrage universel au conseil général, le conseiller général devient sans doute un des élus les plus légitimes sur un des espaces institutionnels les moins stables et peut-être les moins significatifs : le canton.

Dans cette perspective, et dans la dimension plus géographique du processus de construction territoriale qui nous intéresse, le conseiller général n'a-t-il pas là un moyen inespéré de travailler efficacement à la construction et à la mise en scène à la fois de son territoire d'élection devenu EPCI et de son territoire théorique d'action : le département ? La construction en territoire de ces deux espaces fustigés pour leur manque d'épaisseur, remis sans cesse en cause, construit de surcroît une architecture potentielle qui renforce le hiatus profond entre le monde urbain et le monde rural. En effet, en ville le découpage cantonal ne possède pas le même sens et n'offre pas les mêmes opportunités. Le rural se verrait ainsi renforcé, flattant une fois de plus après la mise en place par l'INSEE du zonage en aire urbaine, ce mythe d'une France rurale telle que J. Giono a pu la décrire ou J. F. Gravier la rêver (B. Marchand, 2001), et faire perdurer l'identité braudélienne de la France.

Ce retour en grâce du département et du canton est pourtant contesté par un discours ambiant et récurrent qui récuse son statut de territoire, réservant 
plutôt ce dernier aux EPCI et aux «pays » qui répondraient mieux à une certaine idée d'un territoire historique et vécu. Nous commencerons donc nécessairement par mettre en scène cette pression qui pèse sur cet échelon en nous questionnant sur sa fin annoncée. Mais nous poursuivrons notre lecture en montrant comment cette institution se révèle, dans ses contradictions, particulièrement efficace dans sa capacité à perdurer et comment ses acteurs continuent sa construction en territoire. Ce premier point doit cerner le contexte dans lequel se trouve le conseiller général et nous permettre de comprendre les mécanismes mis en œuvre pour gérer l'urgence dans laquelle il s'estime être aujourd'hui et l'enjeu que recèle cette fonction. Nous analyserons ensuite le rapport intime entre le périmètre des EPCI et la matrice cantonale pour questionner la nécessité du redécoupage évoqué par le «rapport Mauroy» (2000), suggéré par M. Vanier (2004) et objet d'une proposition de loi déposée au Sénat le 29 mars 2005. Pour finir, nous poserons quelques éléments pour tester notre hypothèse concernant l'enjeu que revêt le cumul de la présidence d'un EPCI et du mandat de conseiller général. On s'attardera aussi sur la difficulté pour le président d'EPCI de briguer un mandat de conseiller général $\mathrm{du}$ fait, c'est une hypothèse, de la faible lisibilité que revêt sa fonction. Au final, ce sont bien les mécanismes de construction des territoires locaux et la puissance du modèle territorial à l'œuvre que nous souhaitons questionner sur cette base intermédiaire représentée par le département et ses cantons.

\section{1- Département et canton : des territoires en sursis ?}

Nous associons ici département et canton car ils sont aujourd'hui intimement liés. Le rôle du canton est essentiellement contenu dans son statut de circonscription électorale pour l'élection des conseillers généraux siégeant au niveau du département. Depuis le début du XXème siècle, le département est en sursis et le canton perd le peu de sens qui lui restait. Pourtant, faibles et menacés, ces deux espaces perdurent et personne n'a pris sur lui de les éliminer. Mieux, les lois de décentralisation de 1982 et 1983 ont confirmé ${ }^{7}$ le statut de collectivité territoriale au département et la loi constitutionnelle de mars 2003 lui donne encore plus de compétences en renforçant son rôle dans la proximité géographique. Nous souhaitons ici mettre en scène ce sursis qui se prolonge, et montrer par là la capacité des acteurs de ces institutions à entrer en résistance, à l'organiser et finalement à renforcer ces espaces, voire à les ériger en territoires.

\section{1- Faiblesses et menaces}

Dans l'histoire des institutions françaises, le département incarne le centralisme de la France et fait donc l'objet de menaces récurrentes depuis le début du XXème siècle. Il est vrai que le législateur n'a eu de cesse d'inciter à la création de territoires (pays et EPCI) qui, sans être ouvertement présentés comme concurrents, sont souvent utilisés pour les affaiblir et participent à leur effacement.

${ }^{7}$ Le département créé en 1789 est reconnu comme collectivité locale par la loi du 10 août1871 (le conseil général est élu au suffrage universel) 


\section{1 - Une image conservatrice}

La présence du département dans les «Lieux de mémoires », l'œuvre dirigée par P. Nora, si bien construite en légitimité par M. Roncayolo (1997), atteste, sinon de son caractère incontournable, du moins de sa pérennité. Il faut souligner le courage scientifique de l'auteur, se posant ici en défenseur d'un objet, certes spatial, mais totalement incongru pour le géographe. Il rappelle dans ce texte que les plus grands géographes ont été sollicités pour pourfendre le département, que P. Foncin dès 1898 présentait comme "pris en tenaille, entre les "pays» considérés comme plus authentiques et les régions, échelle plus moderne de la vie économique et sociale » (cité par M. Roncayolo, 1997, p.2961). Et M. Roncayolo de poursuivre en évoquant le rôle joué par P. Vidal de La Blache en personne dans les projets de lois contemporains de la première guerre, où il est littéralement : "appelé à la rescousse, comme défenseur des arrondissements, plus proches des pays, contre l'organisation départementale » (p.2962).

Né dans les esprits ultras rationnels des révolutionnaires dès le lendemain de la révolution, le département est fondé sur le principe d'égalité doublé de celui d'accessibilité qui a fait arriver jusqu'à nous la fameuse image, érigée en règle de délimitation, de la journée de cheval pour rejoindre le chef-lieu. Il doit aussi garantir l'unité de la France en niant les limites des provinces et de toutes les entités historiques et/ou culturelles susceptibles de réclamer autonomie ou privilèges. Le département garde l'image de la raison pour laquelle il a été créé : donner un cadre légal à la France et des circonscriptions électorales pour élire la future assemblée et récolter les impôts. Cette image perdure en dépit de brillantes démonstrations, notamment celle de M.V. Ozouf-Marignier (1992), sur l'extrême complexité de l'entreprise et la négociation âpre entre les notables des territoires pour respecter au final bien plus de limites culturelles que la froideur de leur naissance ne le laissait présager.

Si l'image de cette institution, pourtant collectivité locale depuis 1871, est celle du centralisme, c'est sans doute aussi le fait de la collusion historique entre « un préfet qui dirige et un conseil général qui régule » (R. Carrier, 2002, p.79). Et cet adage renvoie à l'idée d'une structure conservatrice, propre à privilégier la reproduction des notables par le clientélisme. Cette image est aussi celle du canton, espace fortement associé au département puisqu'il constitue la circonscription électorale des conseillers généraux, là où le clientélisme s'applique.

Or, à propos du canton, G. Sautel et J. L. Harouel (1997), dans l'histoire des institutions, parlent de «circonscription mineure de faible étendue et de peu d'importance dans l'ensemble du système» (p.88). Espace administratif et électoral, le canton est ainsi souvent présenté comme le maillon faible du maillage territorial français, en même temps que le support incontournable du pouvoir des notables. Il a pourtant longtemps été au cœur des projets de recomposition territoriale. La communauté de cantons ${ }^{8}$, le conseil cantonal ${ }^{9}$, la

${ }^{8} 1795$ : la communauté de canton, abolie en $1800 ; 1883$ : projet Lannessan de retour aux communautés de cantons (non abouti). La communauté de cantons a tout de même suscité un ouvrage de J. Morange en 1971 
conférence des maires du canton ${ }^{10}$ restèrent pour la plupart à l'état de projets mais utilisaient le périmètre du canton comme fondement des recompositions territoriales. Cette référence cantonale renvoie à une société ancienne dont on entretient la nostalgie. Une société qui appartient à l'identité d'une France «braudélienne ».

L'image du bourg rural, soleil qui rayonne sur son canton et ses villages disposés selon un schéma christallérien, n'a plus beaucoup de sens aujourd'hui. Dans une société de plus en plus mobile où l'exode urbain remplace désormais l'exode rural, l'image du canton, espace de solidarité, polarisé par son chef-lieu a vécu. Aujourd'hui le bourg ne représente plus pour personne «le monde extérieur dans son entier : l'administration, la justice, le commerce...»(F. Braudel, 1990 [1986], p.158). Les nouveaux arrivants sont des urbains, pour eux l'image du notable conseiller général n'a pas beaucoup de sens et les migrations pendulaires imposées par le travail et les habitudes de consommations et de loisirs effacent le rôle du chef-lieu, voire celui de centralités secondaires qui subissent la concurrence des grandes zones commerciales à la périphérie des métropoles et des villes moyennes. Les limites du canton s'effacent, sa connaissance et sa reconnaissance en tant qu'entité disparaissent.

\section{2- La concurrence des EPCI et des Pays}

Le rapport « refonder l'action publique locale » (P. Mauroy, 2000) pose l'intercommunalité comme menace ultime pour le département. A l'affaiblissement de son rôle, il faut ajouter la liberté offerte aux EPCI de déterminer leur périmètre et de bousculer, éventuellement, les limites tant à l'intérieur du département (cantons, arrondissements) qu'entre les départements eux-mêmes. Cette liberté face aux limites administratives est affichée depuis $1984^{11}$ à la mise en place des chartes intercommunales. G. Novarina et S. Martin notaient que " ni la loi ni le décret ne donnent d'indication quant à la correspondance du périmètre de la charte avec celui de circonscriptions administratives, de cantons, ou quant à ses caractéristiques. »(1985, p.204). Les deux chercheurs analysaient même le vide juridique initiant cette nouvelle procédure comme "un moyen pour les petites communes rurales de se faire entendre directement par le conseil régional sans avoir recours aux intermédiaires habituels que sont les grands notables, députés, sénateurs, membres du bureau du conseil général. »(1985, p.209). Cette opportunité offerte aux petites communes ouvrait la voie aux discours réformateurs qui plaidaient à nouveau pour une simplification de l'organisation territoriale sur la base d'intercommunalités, de pays et de régions, alors même que le département venait d'être reconduit comme collectivité territoriale par les lois de décentralisation.

A une autre échelle, le « pays », créé par la LOADT de 1995, renforcé par la LOADDT de 1999, et simplifié par la loi « urbanisme et habitat » de 2003,

\footnotetext{
${ }^{9} 1821$ le projet Villelle de mise en place d'un conseil cantonal consultatif

${ }^{10} 1837$, le projet Vivien d'une conférence des maires du canton. 1882, le projet Gobbet de doter le canton d'une personnalité administrative

${ }^{11}$ Décret du 26 juin relatif aux chartes intercommunales
} 
est une entité fondée sur une homogénéité (culturelle, géographique ou historique) et dotée d'une capacité à s'autodéterminer. La constance avec laquelle le législateur l'a intégré dans les dernières lois fait montre d'une certaine vivacité du concept/slogan qui va de pair avec le flou qui l'entoure. Sa souplesse plaît et l'échelle, même non avouée, proche de celle d'un arrondissement, correspond à ce que beaucoup considèrent comme étant la bonne dimension : celle des «pays » vidaliens. Certains maires n'hésitent pas à utiliser ces « pays » contre le département, notamment dans les villes petites ou moyennes qui ont réussi à associer, au sein d'un pays, l'aire urbaine et l'espace rural environnant. L'image de l'origine centralisée du département s'oppose à la liberté laissée aux acteurs locaux de déterminer le périmètre de leur pays. Le pays peut donc être un «territoire », avec toute l'épaisseur que la géographie confère à cet objet (G. Di Méo, 1998), doté d'une histoire, de légendes, révélé par les pratiques de ses habitants. Il peut même remettre en cause les frontières, et pourquoi pas, à terme, remplacer le département. C'est sans doute cette idée qui a conduit à la naissance d'un des tout premiers pays reconnus (au titre de la LOADT) : le pays « Pays-Basque $»^{12}$.

La tutelle régionale sur la politique des pays ${ }^{13}$, si elle est conforme à la compétence d'aménagement du territoire de cette institution, ne plaide pas en faveur du département. Le conseil général, en tant qu'institution, est a priori un peu en marge de la démarche, même si le conseiller général, acteur local majeur, est très présent sur le terrain. Cette tutelle apporte une certaine garantie concernant le respect des limites régionales, mais peut, potentiellement, favoriser le dépassement des limites départementales.

Mais au-delà de ces questions de frontières et de structures, la démarche même bouscule la position des notables locaux et notamment celles des conseillers généraux. L'idée d'une gouvernance des territoires avec la mise en place de conseils de développement regroupant les forces vives du pays introduit un rapport de force complexe qui impose un nouveau regard et sans doute des comportements novateurs. A cet égard, le travail réalisé pour dégager le diagnostic du territoire et l'écriture de la charte mettent le territoire en mouvement et renforcent les exigences de ces nouveaux acteurs. Les élus locaux, jusque-là seuls à connaître les dossiers et à maîtriser les procédures, doivent aujourd'hui partager cette connaissance avec d'autres acteurs qui, s'ils n'ont pas la légitimité des urnes, représentent une force considérable du fait de leur mobilisation. Les exigences de ces nouvelles élites sont grandes, à la hauteur du travail fourni, et les élus sont en ligne de mire. Cette nouvelle gouvernance et ses implications constituent sans doute un nœud important des changements potentiels qui se dessinent.

\footnotetext{
${ }^{12}$ le « Pays Basque » est reconnu en décembre 1997

${ }^{13}$ Cette tutelle est particulièrement forte dans certaines régions (Centre). En Aquitaine la politique des PCD (projet collectif de développement) qui devait préfigurer les pays fut peu efficace du moins dans la configuration spatiale des « pays »
} 


\section{2- Mais le département a des ressources}

Pour autant, toutes ces menaces n'ont fait disparaître ni le département, ni le canton. Le rapport Mauroy ne va d'ailleurs pas dans ce sens. Si la question a vraisemblablement été soulevée au sein de la commission, celle-ci a " considéré que la suppression de ce niveau comporterait plus de risques que de bénéfices réels. Le département reste irremplaçable en matière d'action de proximité notamment en ce qui concerne le social, la culture ou les équipements » (P. Mauroy, 2000, p.2). Les lois récentes, comme on l'a déjà vu, ont suivi ces conseils.

D'un autre côté, le conseiller général reste un relais local indispensable et le département est pour cela une échelle particulièrement efficace et le canton, ancrage local du conseiller général, une maille sinon bonne du moins existante pour assurer le relais et la proximité. La décentralisation, tout en conservant ce rôle tacite de proximité au conseiller général, lui a donné une autonomie qui lui permet en plus de construire politiquement son territoire ou plutôt ses territoires : le canton et le département. D'ailleurs, en même temps que les nouvelles entités ont théoriquement vocation à le transcender, elles respectent quasi scrupuleusement ses limites et ne font finalement que légitimer un peu plus la pertinence de son périmètre et, au final, de son institution.

Tout le paradoxe réside dans cette obsolescence affirmée et dans une lisibilité toujours plus importante. En effet, lors des élections régionales les listes sont départementales (même si le mode de scrutin inauguré en 2004 complexifie quelque peu la lecture) et poussent à reconnaitre le département comme une entité lisible qui, pour l'instant, supplante largement la Région en notoriété. Le canton, quant à lui, bénéficie d'une promotion qui pousse encore plus loin l'ambiguïté puisque les élections des conseillers généraux sont dites cantonales. H. Portelli (1996) souligne d'ailleurs que les modes de scrutins concernant ces deux assemblées (Conseil Régional et Conseil général) favorisent de fait un retour des investissements sur le territoire électoral et par là une forme de clientélisme et une logique de «guichet », renforçant la lisibilité et le travail de construction.

Le département a la vie dure. Il suffit d'observer les revendications départementales pour comprendre qu'il s'agit, pour beaucoup d'élus, mais aussi les élites locaels, d'une solution à tous leurs problèmes. Le Pays Basque est l'exemple type de cette revendication qui prend corps dans la rivalité bascobéarnaise et dans le caractère «intolérable » du traitement depuis Pau des affaires basques de compétences départementales. Le Pays Basque n'est d'ailleurs pas isolé dans cette revendication, de nombreux exemples pourraient être présentés pour abonder dans ce sens.

Ces revendications départementales montrent que le département n'est pas mort. Il constitue une valeur refuge que beaucoup d'élus n'hésitent pas à animer, alors même qu'il est fustigé pour son obsolescence. On voit même depuis peu, dans la mouvance des marques de vêtements identitaires fondées sur des appartenances très géographiques, certaines marques utiliser le département $(64,65,33,93)$ comme référence. Quand l'instance économique se saisit de l'affaire, on peut penser que la prophétie lancée par les politiques 
d'ériger en territoire cette entité révolutionnaire est sur la voie de l'autoréalisation.

G. Novarina et S. Martin (1985) expliquent d'ailleurs que finalement, dans la pratique, "là où les réseaux notabiliaires traditionnels sont puissants, conseils généraux et conseils régionaux continueront à saupoudrer, sur tout le territoire, leurs aides. »»(G. Novarina, S. Martin, 1985, p.212). C'est ce clientélisme qui est souvent encore dénoncé par la société civile, ou par les candidats de l'opposition aux élections cantonales, mais c'est également lui qui entretient le pouvoir des notables dont parlent les auteurs et dont P. Grémion (1975) a démonté la puissance des mécanismes de reproduction.

La ressource majeure du département est incarnée par les conseillers généraux. Elus sur la base du canton, ils sont très présents dans le local et savent verrouiller leur circonscription. L'exemple des Pays est à ce titre révélateur. Il suffit de regarder les conseils d'administration de ces nouvelles institutions pour se rendre compte que les conseillers généraux sont omniprésents. Pour une structure dont on dit qu'elle pourrait faire disparaître le département elle semble bien prise en main par les représentants du conseil général. Cette présence témoigne de l'importance qu'accordent les conseillers généraux à ce nouvel outil, et surtout de la nécessité qu'ils ont « d'être dedans » pour voir venir la menace. Et ce principe de prise en main semble également se vérifier à l'échelle de l'intercommunalité.

\section{2- La communauté de communes cantonale comme norme ?}

De proche en proche, on finit par retomber sur le canton, qui redevient un « espace projet» potentiel. Le dictionnaire juridique de J. B. Albertini et all. (1993) dit même que «le canton peut servir de cadre à la coopération intercommunale [...] Il constitue un espace intéressant pour la mise en auvre d'actions de développement local.» (p.62). Cette idée vient sans doute de la position singulière de cette circonscription à la fois territoriale, bien que peu institutionnalisée, et électorale, qui lui confère la mission «d'organiser le voisinage immédiat des unités administratives élémentaires »(A. Bleton-Ruget, 1999, p.37) ${ }^{14}$. Cette lecture peut paraître quelque peu optimiste et J. P. Charrié (1995) se charge de la nuancer en rappelant le clientélisme sous-jacent à ce qu'il appelle les «réflexes cantonaux» (p.100) dans la structuration de l'intercommunalité. Toujours est-il que ces réflexes fonctionnent et finissent par construire une légitimité quasi naturelle. Cette normalisation conduit même J.F. Gaucher et G. Baudelle (1995) à parler de «modèle cantonal ${ }^{15}$. Ils montrent la prévalence de ce modèle en Bretagne avec 50 communautés de communes sur 74 construites sur cette base en 1995.

Ceci interpelle tout de même les attendus du rapport Mauroy (2000) qui posent comme principe de base «l'absence de coüncidence entre bassins de vie

\footnotetext{
${ }^{14} \mathrm{~A}$. Bleton-Ruget cite Pierre Legendre, Histoire de l'administration de 1750 à nos jours, Paris : PUF, 1968

${ }^{15}$ «On considère qu'il y a modèle cantonal de communautés de communes lorsque celles-ci recoupent le périmètre cantonal ou se redessinent aux limites du patron cantonal» (J.F. Gaucher et G. Baudelle, 1995, p.61)
} 
et découpages administratifs [...] Dans de nombreux cas, les découpages administratifs infradépartementaux (arrondissements ou cantons) n'ont plus de réalité au quotidien. En revanche, des solidarités et des coopérations locales voient le jour, en particulier, dans le cadre de l'intercommunalité et de la création des pays, souvent sur la base des bassins de vie ou des bassins d'emploi. Ceci est vrai en particulier des 1849 établissements publics de coopération intercommunale à fiscalité propre qui regroupent 21358 communes. » (P. Mauroy, 2000)

On voit donc un hiatus entre d'un côté des structures intercommunales qui seraient repérées comme très «cantonales », et de l'autre une analyse qui les présente comme de nouveaux espaces de solidarité, propres à remettre en cause les découpages administratifs existants.

\section{incohérente}

\section{1- Quand le canton s'impose comme la solution la moins}

La question ici est bien d'évaluer le succès du canton comme référence dans la construction du périmètre des intercommunalités. En effet, dans l'hypothèse ou les EPCI seraient calqués sur le canton, un redécoupage qui s'appuierait sur la base des structures intercommunales n'a pas de sens. Ce découpage ne serait pas beaucoup plus pertinent si les EPCI ne faisaient que redessiner d'autres cantons sans dépasser la taille de ceux-ci. C'est cette hypothèse que nous allons tester sur les cinq départements que nous avons choisis $^{16}$.

Tester le modèle cantonal d'intercommunalité impose également de rappeler la grande diversité des situations départementales. Cette diversité concerne d'une part le découpage lui-même, d'autre part la politique départementale face à la question intercommunale. Il faut ajouter à cela l'influence des villes, qu'elles soient des métropoles comme Bordeaux, ou des villes moyennes, comme Pau ou le BAB (Bayonne, Anglet, Biarritz), et à un degré moindre Périgueux, Agen ou Mont-de-Marsan. Les villes importantes atténuent l'influence cantonale sur la construction des EPCI du fait de la spécificité du découpage des cantons urbains et périurbains. Cette considération nous a d'ailleurs poussé à exclure la Gironde de notre échantillon.

\footnotetext{
${ }^{16}$ Nous avons choisi quatre départements aquitains (l'exclusion de la Gironde est liée à l'influence de la métropole bordelaise) plus la Corrèze, département sur lequel nous avons pu travailler dans le cadre de l'observatoire de l'A89.
} 
Tableau $n^{\circ} \mathbf{1}$ : communautés de communes et limites cantonales en 2004

\begin{tabular}{|c|c|c|c|c|c|c|}
\hline & $\begin{array}{l}\mathrm{Nb} \\
\mathrm{d}^{\prime} \mathrm{EPCI}\end{array}$ & \multicolumn{5}{|c|}{ EPCI=canton } \\
\hline & & égal & + ou -1 commune & $+\mathrm{ou}-2$ communes & total & $\%$ \\
\hline $\begin{array}{l}\text { Pyrénées- } \\
\text { Atlantiques }\end{array}$ & 28 & 6 & 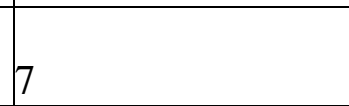 & 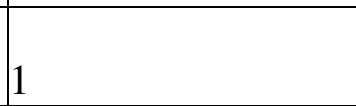 & 14 & 50,00 \\
\hline Landes & 22 & 8 & 7 & 1 & 16 & 72,73 \\
\hline $\begin{array}{l}\text { Lot-et- } \\
\text { Garonne }\end{array}$ & 21 & 3 & 1 & 1 & 5 & 23,81 \\
\hline Dordogne & 52 & 5 & 7 & 1 & 13 & 25,00 \\
\hline Corrèze & 23 & 6 & 1 & 3 & 10 & 43,48 \\
\hline & 146 & 28 & 23 & 7 & 58 & 39,73 \\
\hline
\end{tabular}

Nous avons considéré que le modèle cantonal pouvait s'accommoder de quelques entorses marginales de plus ou moins 2 communes du canton dans ou hors de l'EPCI. Cette latitude permet de régler le jeu des conflits locaux classiques souvent fondés sur des histoires locales de personnes.

Les chiffres sont moins impressionnants que ceux évoqués par J.F. Gaucher et G. Baudelle pour la Bretagne. Avec une moyenne de 40\%, le modèle cantonal n'est pas écrasant. Si les Landes et à un degré moindre les Pyrénées-Atlantiques voire la Corrèze reproduisent assez bien la matrice cantonale dans la structuration intercommunale, la Dordogne et le Lot-etGaronne ne semblent pas fonctionner sur ce schéma. Ce bilan comptable permet-il pour autant de conclure que la remise en cause très partielle et localisée de la matrice cantonale ouvre a un redécoupage possible, plus proche de territoires plus cohérents, fondé sur les EPCI ? Les EPCI non cantonaux correspondent-ils à de nouveaux espaces de solidarité englobants? Si on observe d'un peu plus près les 73 EPCI qui ne respectent pas les limites cantonales on se rend compte que la réalité d'une recomposition sur les bases de nouvelles solidarités est très limitée.

Si on considère la faible pertinence des limites départementales, les EPCI devraient pouvoir s'en affranchir et se calquer sur les bassins de vie et de solidarités qui transgressent aujourd'hui largement ces cadres. La loi l'autorise, même si on ne peut pas dire qu'elle l'encourage. Or, dans notre échantillon, seules 3 communautés de communes sont dans ce cas. Deux d'entre elles franchissent même les limites régionales. Ce faible nombre semble suspect et encore, parmi ces trois expériences, une seule semble réellement s'appuyer sur une base suffisamment large pour prétendre proposer un projet novateur.

Au-delà du dépassement des limites, on peut considérer que pour constituer de véritables bassins de solidarité, il est indispensable que les EPCI bouleversent radicalement la matrice cantonale. Les cantons, souvent très petits et regroupant très peu de communes et parfois très peu d'habitants, ne constituent plus aujourd'hui un découpage crédible pour une intercommunalité performante. Or une observation un peu fine des 70 EPCI concernés révèle que la plupart d'entre eux sont soit à l'intérieur même d'un canton, donc plus petit que l'espace administratif existant, soit recoupent les limites cantonales mais 
reproduisent un espace de la taille approximative des cantons du département. Ces deux cas de figures ne peuvent raisonnablement pas être considérés comme de nouveaux espaces de solidarités.

Combien d'EPCI s'avèrent finalement intéressants dans cette perspective?

Tableau $\mathbf{n}^{\circ} \mathbf{2}$ : Les communautés de communes qui dépassent les limites et la taille du canton

\begin{tabular}{|l|l|l|}
\hline & \multicolumn{2}{|l|}{$\begin{array}{l}\text { Nombre de communautés de communes dépassant les limites et la } \\
\text { taille d'un canton du département }\end{array}$} \\
\hline Dordogne & 2 & $\begin{array}{l}\text { Ces deux communautés de communes restent néanmoins de } \\
\text { petite taille (14 communes). }\end{array}$ \\
\hline Corrèze & 2 & $\begin{array}{l}\text { Une de ces deux communautés est marquée par l'influence } \\
\text { urbaine de Tulle. }\end{array}$ \\
\hline Lot-et-Garonne & 7 & $\begin{array}{l}\text { C'est le département qui autorise le plus la transgression du } \\
\text { modèle cantonal avec quelques EPCI très vastes (plus de 20 } \\
\text { communes) alors que le nombre moyen de communes par } \\
\text { canton est parmi les plus faibles (7,95) }\end{array}$ \\
\hline & 4 & $\begin{array}{l}\text { Une de ces communautés est marquée par l'influence urbaine } \\
\text { d'Oloron-Sainte-Marie, les trois autres regroupent } \\
\text { partiellement ou en totalité des communes de montagne. A } \\
\text { noter que certains EPCI cantonaux regroupent beaucoup de } \\
\text { communes (parfois plus de 20) mais sur un seul canton très } \\
\text { vaste. C'est notamment le cas dans le Nord du département. }\end{array}$ \\
\hline Pyrénées-Atlantiques & & $\begin{array}{l}\text { Une de ces communautés est marquée par l'influence urbaine } \\
\text { de Dax. A noter que le nombre de communes par EPCI est } \\
\text { très inégal du fait d'une grande différence dans la superficie } \\
\text { des communes entre le Nord et le Sud du département. }\end{array}$ \\
\hline Landes & 2 & $\begin{array}{l}\text { Ces 17 EPCI représentent 11,64\% des 146 communautés de } \\
\text { communes des 5 départements }\end{array}$ \\
\hline TOTAL & 17 & \\
\hline
\end{tabular}

Le tableau $\mathrm{n}^{\circ} 2$ révèle que $11,64 \%$ des communautés de communes seulement sont réellement plus vastes qu'un canton et bouleversent ses limites. On peut d'ailleurs noter que le Lot-et-Garonne est une exception dans le paysage intercommunal local puisqu'il représente à lui seul plus de $40 \%$ de ces cas de figure. Ces chiffres sont significatifs du fait que lorsqu'ils ne se calquent pas sur les limites du canton, les EPCI dessinent rarement de nouveaux espaces de solidarité plus vastes et plus cohérents sur lesquels pourrait s'appuyer un nouveau découpage. On est plutôt dans une reconfiguration d'intercommunalités restreintes qui n'atteignent pas une masse critique suffisante. Le cas du Lot-et-Garonne laisse tout de même entrevoir la possibilité d'un fonctionnement autre, mais, même dans ce cas, l'innovation reste limitée 
car on peut se demander dans quelle mesure la petite taille des cantons ne rend pas ce dépassement nécessaire.

\section{2- Le jeu politique du conseiller général}

Dans ce contexte, le conseiller général peut devenir l'homme de la situation en milieu rural. Il détient son mandat sur un territoire supra communal situé à l'échelle intermédiaire, entre le Pays et la commune. Une échelle qui est reconnue comme une nouvelle maille possible ; une échelle adoptée par bon nombre de coopérations intercommunales, on vient de le voir, le plus souvent à l'initiative du conseiller général lui-même et avec le soutien de l'institution départementale. On retrouve ici le jeu des acteurs locaux et des élus qui renvoie à l'analyse de Cl. Sorbets (1993, p.38) :

"Celui-ci (l'élu local) en jouant alternativement un rôle politique et un rôle apolitique, selon qu'il se trouvera jouer au centre ou à la base, fonde sa légitimité à gouverner et à assumer son rôle de notable [...] sur la capacité à ruser : à ruser avec sa base et ses représentations comme avec le centre et son appareillage[...]. Vis-à-vis de la première, il cherchera à intervenir pour ses mandants, vis-à-vis de la seconde, il votera des réglementations générales au centre et en cherchera les possibilités d'y déroger pour sa base d'élection »

Mais ce jeu se spatialise singulièrement, se territorialise même, avec le centre, représenté ici par le conseil général, qui devient complice de la ruse, agissant un peu comme une périphérie en danger qui cherche d'abord à pérenniser son existence. Il accompagne de sa politique la maîtrise des EPCI afin de préserver son unité et son autorité. Ainsi, la politique départementale devient un défi permanent de construction territoriale à la fois dans ses limites extérieures et dans ses découpages intérieurs. Tout cela n'est pas nouveau et concerne toutes les collectivités locales, de la commune à la région mais la construction interne prend ici des modalités particulières autour de ces cantons, territoires d'élection, érigés en territoires d'action dont la construction peut commencer, et ces pays, investis par les conseillers généraux, qui épousent la forme d'arrondissements, actuels ou en devenir.

Nous ne pouvons pas prétendre ici présenter un modèle unique notamment parce que le conseil général lui-même, par sa politique, va orienter les choix et favoriser ou non ces cumuls. C'est d'ailleurs peut-être dans ce domaine qu'il possède une marge de manœuvre politique difficile à trouver ailleurs. Mais force est de constater que les départements choisis, à l'exception notable du Lot-et-Garonne, vérifient relativement bien nos hypothèses. Derrière des pratiques et des stratégies, on trouve également de belles histoires de fidélité, des positions cantonales qui prennent naissance dans le respect de la figure du notable et dans le respect du «territoire administratif » tel qu'il est imposé par l'histoire depuis la révolution.

Déjouant toutes les prophéties et toutes les analyses, la tendance mise en lumière dans cette partie conduirait à faire du canton, tant dans le gabarit que, à un degré moindre, dans ses limites actuelles, une forme d'optimum dimensionnel pour la communauté de communes rurale. Cet optimum mythique (L. Ortiz 1994) deviendrait une réalité par le truchement d'un discours récurrent et incantatoire de l'acteur politique local dont l'effet performatif (J. L. Austin, 
1991 [1962]) semble se vérifier ici. Ce discours crée l'objet, induit des représentations voire des comportements nouveaux que l'on peut considérer au final comme participant à la réalisation de la prophétie scandée par le discours (R. K. Merton, 1997 [1953], P. Watzlawick, 1996 [1981], J. F. Staszak 2000, M. Vanier, 2004). Le discours ambiant qui évoque la possibilité de créer de nouvelles circonscriptions pour élire les conseillers généraux sur la base de territoires rénovés fondés sur les découpages intercommunaux participe de cette prophétie car il semble que tenir compte des intercommunalités, signifie aujourd'hui, au moins pour une bonne moitié, se rapprocher finalement du canton. Et si le conseiller général est aussi président de l'EPCI, la boucle est bouclée...

\section{3- Conseiller général - président d'EPCI : le télescopage des compétences}

Le cumul entre le mandat de conseiller général et celui de président de structure intercommunale, voire de Pays n'est pas une stratégie nouvelle. Elle avait déjà été mise en lumière par G. Novarina et S. Martin (1985).

L'importance grandissante des EPCI du fait des compétences accrues qui leur sont transférées et des budgets qui en découlent donne une actualité particulière à cette remarque. Et dans le contexte de crise du politique qui marque l'époque actuelle, la légitimité démocratique des acteurs est particulièrement sensible et touche l'univers novateur des EPCI (M.-Ch. Bernard-Gelabert, 1997). Ce débat s'est amplifié depuis 1999, avec l'évocation du caractère inévitable d'un passage au suffrage universel. Dans ce contexte, le cumul du statut de conseiller général et de président de l'EPCI, peut être lu comme une solution «naturelle » à la question de la légitimité du président de la structure et comme un surcroît d'efficacité pour les deux fonctions. Pour autant ce cumul stratégique crée une multitude de confusions, tant au niveau du discours qu'au niveau de l'espace de référence des deux fonctions.

Les élections cantonales de 2004 nous ont permis d'analyser d'un peu plus près ces pratiques et ces stratégies spatiales en construction.

\section{1- Un cumul relativement classique}

Tableau $\mathbf{n}^{\circ} \mathbf{3}$ : le cumul de la présidence d'une communauté de communes et du mandat de conseiller général avant et après les élections cantonales de 2004

\begin{tabular}{|l|l|l|l|l|}
\hline & $\begin{array}{l}\text { Nombre } \\
\text { d'EPCI }\end{array}$ & $\begin{array}{l}\text { Nombre de } \\
\text { Cantons }\end{array}$ & $\begin{array}{l}\text { Conseiller général président } \\
\text { de communauté de communes } \\
\text { avant 2004 }\end{array}$ & $\begin{array}{l}\text { Conseiller général président } \\
\text { de communauté de communes } \\
\text { après 2004 }\end{array}$ \\
\hline Dordogne & 53 & 52 & $14(26,4 \%)$ & $12(22,6 \%)$ \\
\hline Landes & 23 & 33 & $11(47,8 \%)$ & $11(47,8 \%)$ \\
\hline Pyrénées-Atlantiques & 30 & 58 & $8(26,7 \%)$ & $9(30 \%)$ \\
\hline Corrèze & 24 & 35 & $11(45,8)$ & $9(37,5 \%)$ \\
\hline Lot-et-Garonne & 21 & 42 & $5(23,8 \%)$ & $4(19,04)$ \\
\hline Total & 151 & 220 & $49(32,45 \%)$ & $45(29,8)$ \\
\hline
\end{tabular}


La figure du notable à la fois président d'EPCI et conseiller général est relativement classique. Dans les cinq départements tests, on trouve 49 « cumulards » avant les élections de 2004 et 45 après, ce qui représente près de $30 \%$ des présidents d'EPCI. Tous les départements ne reprennent pas le modèle puisque le Lot-et-Garonne est en retrait, avec seulement 4 cas recensés. Ce résultat n'est pas étonnant puisque ce département était déjà en retrait sur le respect du découpage cantonal dans les délimitations d'EPCI. Or, le cumul est particulièrement intéressant lorsque le canton est totalement inclus dans l'EPCI pour pouvoir diriger les retombées issues des actions menées par la structure intercommunale, l'idéal-type étant l'EPCI strictement cantonal. C'est d'ailleurs dans les deux départements qui respectent le mieux le modèle cantonal, les Landes et la Corrèze, que le cumul est le plus fréquent (relativement au nombre d'EPCI). Les Pyrénées-Atlantiques se situent entre les deux avec un ratio un peu plus faible mais une valeur absolue équivalente, tout comme la Dordogne, dont l'éclatement intercommunal fait baisser le ratio, mais qui constitue la valeur absolue la plus forte.

Ces chiffres sont intéressants, mais si on peut dire que ce type de cumul est classique, il ne représente pas réellement une norme. En revanche, les élections cantonales de 2004 nous permettent de voir que ce statut dont l'intérêt s'est considérablement accru, est recherché.

\section{quiétude des anciens}

32- La difficile ascension des nouveaux notables et la

Au-delà de l'importance relative de ce modèle de cumul, il est en effet intéressant de se demander, au lendemain des élections cantonales de 2004, d'une part si les présidents de communauté de communes se sont lancés dans la bataille électorale et d'autre part s'ils ont connu la réussite. Nous formulons ici la double hypothèse que leur présence dans la campagne témoigne de la prise de conscience de l'intérêt de cumuler les fonctions et que leur réussite ou leur échec peut être considéré comme un révélateur de la lisibilité de ces structures auprès des populations. Bien entendu, il ne s'agit ici que d'affiner quelques hypothèses à partir de l'analyse des cinq départements que nous avons choisis.

Tableau $\mathbf{n}^{\circ} 4$ : Les présidents d'EPCI dans la "bataille" des élections cantonales en 2004

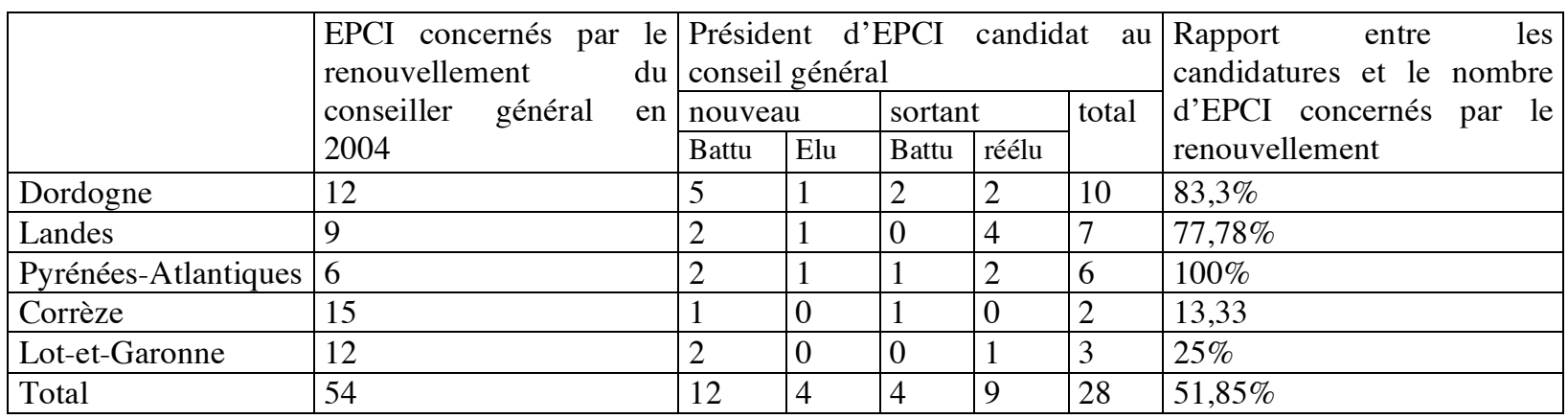


L'intérêt est ici de mettre en évidence le rapport entre le nombre de présidents d'EPCI qui ont brigué le mandat de conseiller général et le nombre d'EPCI concernés par le renouvellement. Les chiffres sont éloquents avec trois départements qui présentent des pourcentages impressionnants puisque dans les Pyrénées-Atlantiques, tous les présidents de communautés de communes qui étaient concernés par l'élection ont brigué le mandat. La Dordogne avec 10 présidents sur 12 et les Landes (7 sur 9) sont également très touchés par le phénomène. Si on peut expliquer encore une fois l'exception lot et garonnaise, la mise en retrait de la Corrèze peut surprendre. Ces chiffres tendent donc à montrer que les présidents d'EPCI ont tenté de saisir l'opportunité d'un cumul qui offre la légitimité des urnes à ceux qui ont la capacité d'action.

Mais cette ascension vers le statut de notable n'est pas facile. P. A. Landel (2004) reprenant l'analyse de Michel Bussi dans le journal Libération du 2 mars 2004, montre d'ailleurs que l'aventure est globalement difficile puisqu'il souligne que «de 1970 à 1992, le pourcentage de conseillers généraux réélus varie de $60 \%$ à $90 \%$ » (p.75). Là aussi, localement, les chiffres sont sans appel. Sur les 16 présidents candidats qui n'étaient pas déjà conseiller général, seuls 4 (soit un quart) ont réussi leur pari. Et encore faut-il relativiser la portée de ces victoires puisque sur ces quatre vainqueurs seul Thierry Boide, pour le canton de Villefranche-de-Lonchat, s'est vraiment imposé à contre courant de la vague socialiste et contre un « sortant» d'envergure.

Plusieurs hypothèses peuvent expliquer la difficulté du président d'EPCI à être élu. Nous privilégierons celle de la faiblesse de la reconnaissance de sa fonction voire l'ignorance même de son existence. On peut penser aussi que lorsqu'il représente la commune-centre il ne jouit pas forcément d'une bonne image dans les communes périphériques. A l'opposé, le conseiller général installé bénéficie des retombées du clientélisme déjà évoqué et de la pérennisation de son statut de notable. Certes on ne connaît pas vraiment son rôle ni ses compétences, mais il représente le canton au niveau du département, et on a du département une représentation assez claire qu'il a participé à construire par son travail quotidien.

L'avantage du cumul des deux fonctions avant les élections est assez évident. La faible marge de manœuvre du conseiller général est compensée par les compétences qu'il détient de sa fonction de président d'EPCI. Et les résultats des conseillers généraux qui cumulaient les deux fonctions avant les élections de 2004 sont inversés par rapport à ceux que l'on vient de voir. Sur 13 notables installés seuls 4 ont été battus (soit $30 \%$ ).

Cette quête de cumul pose tout de même un problème épineux. Si on pose l'hypothèse que la logique qui préside à ce cumul réside essentiellement dans l'efficacité de l'association des deux mandats, alors, on peut s'interroger sur la situation qui découle d'une défaite qui va mettre face à face, sur le même espace ou presque, un président d'EPCI et un conseiller général qui viennent de s'opposer lors de l'élection. Si le cumul peut donner de l'efficacité aux deux fonctions, l'opposition et peut-être l'affrontement, peuvent aussi ralentir le processus de développement. Travailler de plus près ces structures serait un bon moyen de comprendre la marge de manœuvre de chacun des acteurs. 
Il semble acquis en revanche que le cumul va permettre de lancer la « fabrique du territoire » intercommunal et donc cantonal et au-delà du territoire départemental et finalement pourquoi pas du «pays ». Le conseiller général va pouvoir disposer d'outils pour agir et d'outils pour communiquer. La prophétie territoriale trouve ici un nouveau souffle et son autoréalisation ne semble plus faire de doute.

\section{Pour conclure}

Le département n'est pas mort, et s'il est fragile, sa construction en tant que territoire approprié pas ses habitants est encore à l'œuvre. Il a pour lui deux cents ans d'histoire et presque autant de présence dans les livres d'école. Combien de collectivités peuvent se targuer d'une telle épaisseur historique ? Même les « pays vidaliens », que l'on s'évertue à réinventer à travers les «pays LOADT », ne font que rarement l'unanimité que ce soit pour ce qui est du périmètre ou des origines. Les «pays », remis au goût du jour par les géographes du début du XXème siècle et redécouverts dans le cadre même du département (M. Roncayolo, 1997), se calquent finalement sur les limites départementales voire, à l'intérieur de celles-ci, sur les arrondissements. La veille minutieuse exercée par les conseillers généraux sur ces objets redécouverts finit par les rendre inoffensifs pour la structure départementale. D'autres études en cours d'achèvement, montrent même que l'on peut leur attribuer un rôle dans la conscience territoriale départementale. Le Pays du Périgord Noir que ce soit par son nom ou son logo semble entrer dans cette catégorie.

$\mathrm{Au}$ niveau du canton, le travail du conseiller général est tout aussi remarquable. Moteur dans la construction des EPCI, il est parvenu à faire du canton le périmètre sinon exclusif du moins privilégié, de la coopération intercommunale en milieu rural voire périurbain. Si cet état de fait ne constitue pas en soi une hérésie, le modèle d'un EPCI dont le président est également conseiller général, construit une architecture départementale relativement verrouillée. Le conseiller général du canton transforme son espace d'élection en espace d'action et donne à la fonction de conseiller général une réelle capacité d'action locale. Ces compétences multiples remises entre les mains du même homme doublement légitimé, décuplent la capacité de ce territoire à organiser son développement de façon cohérente. Pour notre propos, ce cumul tend également à favoriser une association d'image entre le «canton-EPCI » d'une part et le conseil général d'autre part. Enfin, on peut faire une lecture pessimiste, réaliste diront certains, en considérant qu'elle décuple aussi le risque de dérives clientélistes que tout le monde s'accorde à dénoncer et organise une confusion d'image et de compétences qui nuit à la lisibilité et à la transparence qui font déjà largement défaut au politique.

On peut néanmoins se demander ce qu'il adviendra des territoires sur lesquels le président de l'EPCI a échoué, et le cas est fréquent, dans sa quête du siège de conseiller général. La disjonction des fonctions peut-elle entraîner une situation de blocage? Pour le dire autrement, les territoires pour lesquels les 
fonctions sont cumulées bénéficient-ils d'une rente relative dans le processus de développement local et dans celui de la construction territoriale ?

Mais, au-delà de ces questions qui demeurent et qui constituent autant de pistes à creuser pour des recherches futures, nous voulons faire ressortir de cette recherche l'extraordinaire prégnance du modèle territorial dans le fonctionnement des acteurs publics et notamment ici des élus. Il est surprenant de voir comment dans une société de la mobilité, au sein de laquelle les individus mettent en œuvre des stratégies réticulaires toujours plus complexes et toujours plus inventives, une frange de cette société, parmi ses élites, continue à ne mobiliser, dans la sphère publique, qu'une figure possible de rapport à l'espace : le territoire. «L'interterritorialité » proposée par M. Vanier au colloque de Rennes «espaces et sociétés aujourd'hui », constitue-t-elle une alternative au «tout territoire» ou au «tout réseau» qui permettrait de mobiliser les deux logiques dans ce qu'elles apportent de plus efficace?

\section{Bibliographie}

Albertini Jean-Benoît, Berenguer Christian et Marx Jean-Luc (1993), Pouvoirs locaux, dictionnaire juridique, Paris : Dalloz, 534p.

Augé Marc (1992), Non-lieux, introduction à une anthropologie de la surmodernité. Paris, Édition du Seuil, 149p.

Austin John L. (1991 [1962]), Quand dire c'est faire, Paris : Seuil, édition anglaise 1962, traduction, 1970, 208p.

Badie Bertrand (1995), La fin des territoires, essai sur le désordre international et sur l'utilité sociale du respect, Paris : Fayard, 276p.

Balligand Jean-Pierre et Maquart Daniel (1990), La fin du territoire jacobin, Paris : Albin Michel, 221p.

Bernard-Gelabert Marie-Christine (1997) Intercommunalité et intercommunalités, in R. Le Saout dir., L'intercommunalité, logiques nationales et enjeux locaux, Rennes : PUR, p.109-122

Bleton-Ruget Annie (1999), « Le canton rural à l'époque révolutionnaire : l'expérimentation du territoire ", in actes des IVème journées d'études historiques de l'université d'Orléans, l'administration territoriale sous la Révolution française, p.37-51

Braudel Fernand (1990 [1986]), L’identité de la France, Vol. 1, Paris : Flamarion, 2ème édition, 410p.

Carrier Renaud (2002), « Préfet et conseil général de l'an VIII à la loi du 10 août 1871 : un siècle de collaboration active », in J. P. Allinne et R. Carrier dir., Préfet et développement local, , Paris : Dalloz, p.77-88

Charrié Jean-Paul (1995), «Intercommunalité à vocation économique : bassin de vie et pays en Aquitaine », in G. Baudelle dir., De l'intercommunalité au pays, Paris : éditions de l'Aube/IAAT, p.97-111

Di Méo Guy (1998), Géographie sociale et territoire, Paris : Nathan Université, 320p.

Gaucher Jean-François et Baudelle Guy (1995), «Les communautés de communes en Bretagne : conceptions territoriales et logiques politiques », in G. 
Baudelle dir., De l'intercommunalité au pays, Paris : éditions de l'Aube/IAAT, p.61-70

Gremion Pierre, 1976, Le pouvoir périphérique. Bureaucrates et notables dans le système politique français, Paris : le Seuil, 478p.

Landel Pierre-Antoine (2004). "Le département et la démocratie locale : la fin des fiefs ?" Pouvoirs locaux, v.3, n62. pp.74-78.

Laurent Loeiz (2002), La fin des départements, le recours aux pays, Rennes : PUR, 151p.

Le Saout Rémy et Madoré François (dir.) (2004). Les effets de l'intercommunalité. Rennes: Presses universitaires de Rennes. 224p.

Lévy Jacques et Lussault Michel (dir.) (2003), Dictionnaire de la géographie et de l'espace des sociétés, Paris : Belin, 1033p.

Lipovetsky Gilles (2004), Les Temps hypermodernes, Paris : Grasset, $186 \mathrm{p}$.

Lyotard Jean-François (1979), La condition postmoderne, Paris : Éd. de minuit, 109p

Marchand Bernard (2001), «La haine de la ville : «Paris et le désert français » de Jean-François Gravier », L’information géographique, n³, p.234253

Mauroy Pierre pres. (2000), Refonder l'action publique locale, rapport au premier ministre de la commission pour l'avenir de la décentralisation, 187p.

Merton Robert K. (1997 [1953]), Eléments de théorie et de méthode sociologique, Paris : Armand Colin, édition américaine 1953, traduction et adaptation Mendras H. 1965, 384 p.

Novarina Gilles et Martin Samuel (1985), La décentralisation, décentralisation et intercommunalité, Paris : Syros/Alternatives, 250p.

Ortiz Laure (1994), «Espace et efficacité de l'action, le mythe de l'optimum dimensionnel », in J. Cl. Némery dir., le renouveau de l'aménagement du territoire en France et en Europe, Paris : Economica, p.183200.

Ozouf-Marignier Marie-Vic (1992), La formation des départements, la représentation du territoire français à la fin du XVIIIè siècle, Paris : EHESS, $363 \mathrm{p}$.

Portelli Hugues (1996), «Les élus locaux : image dans l'opinion et attentes des acteurs », in La décentralisation en France, l'état des politiques publiques, la dynamique des réformes locales, la dimension européenne, Institut de la décentralisation, Paris : La Découverte, p.177-183

Roncayolo Marcel (1997) «Le département», in P. Nora dir., Les lieux de mémoire, Paris : Gallimard, Coll. Quarto, tome 2, pp.2937-2974

Sautel Gérard et Harouel Jean-Louis (1997), Histoire des institutions publiques depuis la Révolution française, Paris : Dalloz, 522p.

Sorbets Claude (1993), "Le mot et la chose », in Mabileau A. dir., A la recherche du local, Paris : L'Harmattan, p.29-40.

Staszak Jean-François (2000), «Prophéties autoréalisatrices et géographie », Espace géographique, n², pp.105-119 
Tesson Frédéric (1996), Les réseaux de villes en France, recherche sur le rapport de l'élu local à l'espace, thèse de doctorat, université de Pau et des Pays de l'Adour, 399p.

Vanier Martin (1995), «La petite fabrique de territoires en RhôneAlpes : acteurs, mythes et pratiques », Revue géographique de Lyon, Vol. 70, $\mathrm{n}^{\circ} 2$, p. $93-114$

Watzlawick Paul (1996 [1981]), «Les prédictions qui se vérifient d'ellesmêmes », in Watzlawick Paul dir., L'invention de la réalité, comment savons nous ce que nous croyons savoir? contribution au constructivisme, Paris: Seuil, édition allemande 1981 traduction 1988, Xème édition, p.109-130. 